\title{
Bloch oscillations in one-dimensional spinor gas
}

\author{
D.M. Gangardt ${ }^{1}$ and A. Kamenev ${ }^{2}$ \\ ${ }^{1}$ School of Physics and Astronomy, University of Birmingham, Edgbaston, Birmingham, B15 2TT, UK \\ 2 School of Physics and Astronomy, University of Minnesota, Minneapolis, MN 55455
}

(Dated: November 1, 2018)

\begin{abstract}
A force applied to a spin-flipped particle in a one-dimensional spinor gas may lead to Bloch oscillations of particle's position and velocity. The existence of Bloch oscillations crucially depends on the viscous friction force exerted by the rest of the gas on the spin excitation. We evaluate the friction in terms of the quantum fluid parameters. In particular, we show that the friction is absent for integrable cases, such as $S U(2)$ symmetric gas of bosons or fermions. For small deviations from the exact integrability the friction is very weak, opening the possibility to observe Bloch oscillations.
\end{abstract}

PACS numbers: 05.30.Jp, 03.75.Kk, 03.75.Mn

Dynamics of ultracold atomic gases with internal (spinor) degree of freedom has been a focus of a number of recent experiments [1]. The observed collective phenomena have revived interest in earlier theoretical works [2] on spin waves in helium and opened a possibility to study nonequilibrium dynamics of quantum liquids.

Due to an unprecedented degree of experimental control it is possible to excite a few atoms into a different hyperfine internal state [3]. This leads to effective spin excitations which may be regarded as impurities moving through the quantum liquid formed by the majority spins. A similar setup was investigated in the context of $\mathrm{He}^{3}$ and $\mathrm{He}^{4}$ mixtures [4, 5]. It was realized that an external particle is "dressed" to form a collective excitation which energy-momentum relation at small momenta $P$ is quadratic $\varepsilon(P) \approx \mu_{d}+P^{2} / 2 M^{*}$. The correlations manifest themselves in quasiparticle effective mass $M^{*}$ being different from the bare mass $M$, as well as in the friction exerted on the quasiparticle by the rest of the liquid.

The collective nature of the excitations is especially apparent in one-dimensional (1D) systems where the strong effects of interactions beyond mean field were recently observed in experiments with cold atoms 6]. In addition to the strong mass renormalization [7], power law behavior of responce functions [8, 9, 10], the dispersion relation of the excitations $\varepsilon(P)$ was shown [9, 11] to be strongly modified by the interactions: parabolic at small $P$, it is actually a periodic function of the momentum with the period $2 \pi \hbar n$, see Fig. 1 Here $n$ is a $1 \mathrm{D}$ density of the gas and periodicity stems from the fact that total momentum $P_{\text {tot }}=2 \pi \hbar n$ can be transferred to the gas as a whole at no energy cost in thermodynamic limit. The periodicity of the dispersion relation drastically affects the dynamics of spin excitations under an influence of the external gravitational force $F$, which becomes uncompensated if the hyperfine state of impurity atoms is insensitive to the magnetic field of the trap [12]. Indeed, momentum of impurity evolves according to $\dot{P}=F$ and its velocity $V=\partial \varepsilon(P) / \partial P$ is a periodic function of momentum and thus exhibits Bloch oscillations. Bloch oscillations are usually associated with an accelerated quantum par-

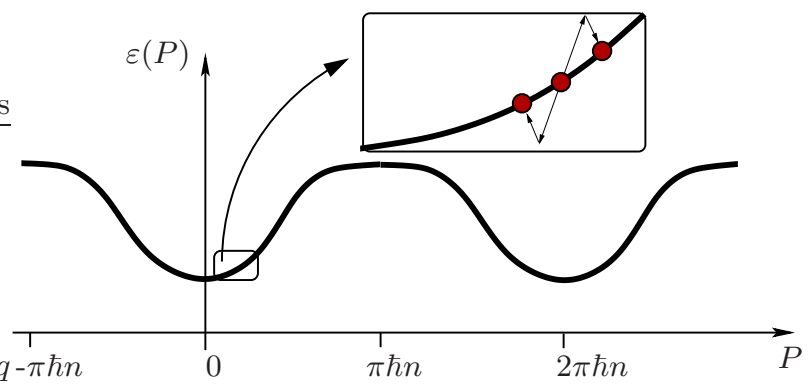

FIG. 1: Dispersion relation of a spin excitation in 1D Bose liquid. Inset: two-phonon processes leading to a dissipation. The arrows, with the slope given by the sound velocity $c$, represent absorption and emission of long wavelength phonons.

ticle in presence of a static periodic potential, see, e.g., Ref. 13] for a recent experimental realization. However, the existence of Bloch oscillations in 1D spinor condensates does not rely on a presence of an external periodic potential. It is the $1 \mathrm{D}$ quantum liquid itself that provides a quasi-periodic potential with the lattice spacing $n^{-1}$ and thus $2 \pi \hbar n$ reciprocal vector.

Contrary to a static periodic potential, a 1D quantum liquid exhibits quantum and thermal fluctuations. Because of the latter the spin excitations with periodic dispersion relation are also subject to a dissipation. A possibility to observe Bloch oscillations depends crucially on the strength of such a dissipation, i.e. the friction force exerted on the spin-flipped particle by the quantum liquid. Indeed, in a presence of friction the equations of motion for the spin excitation take the form

$$
\dot{P}=F-\kappa V ; \quad V=\dot{X}=\partial \varepsilon / \partial P,
$$

where $\kappa=\kappa(V)$ is the coefficient of viscous friction 14]. Consider, e.g., the strong coupling limit where $\varepsilon(P) \approx \mu_{d}+\left(2 \hbar^{2} n^{2} / M^{*}\right) \sin ^{2}(P / 2 \hbar n)[9]$ and $\kappa \approx$ const. Integrating Eqs. (1), one finds for the drift velocity:

$$
V_{\text {drift }}=\kappa^{-1} \begin{cases}F ; & F<F_{c}, \\ F-\sqrt{F^{2}-F_{c}^{2}} ; & F>F_{c}\end{cases}
$$


where the critical force is $F_{c}=\kappa \hbar n / M^{*}$. As a result the drift velocity is a non-monotonic function of the applied force and friction $\kappa$. As discussed below, the latter depends strongly on the temperature and interaction parameters, leading to a non-monotonic dependence of $V_{\text {drift }}$ on them too. In the strong forcing regime, $F>F_{c}$, the drift motion is superimposed with the Bloch oscillations with the period $\Delta T=2 \pi \hbar n / \sqrt{F^{2}-F_{c}^{2}}$. Notice that both the period and the amplitude of the Bloch oscillations diverge as $F$ approaches $F_{c}$ frome above.

To find whether the Bloch oscillations regime is experimentally accessible, one needs to have a detailed understanding of the friction $\kappa(V)$. The friction originates from the interactions between the spin-excitation and the density excitations (phonons) of the rest of the gas. Since the latter propagate with the speed of sound $c$ typically much larger than velocity of the particle $V$, the singlephonon processes do not lead to dissipation according to the Landau criterion [4]. However, the two-phonon processes (see inset in Fig. 11) do lead to energy and momentum relaxation which scales as $T^{7}$ in 3D [4, 5] and as $T^{4}$ in 1D [15] (at small temperature and $V<c$ ). In this paper we evaluate the magnitude of $T^{4}$ friction and express it in terms of "Bose liquid" parameters.

Dimensional arguments [15] lead to $\kappa \sim T^{4} /\left(c^{4} \hbar^{3} n^{2}\right)$, which for typical experimental parameters would make the critical force $F_{c}$ rather large (see below). Fortunately the actual friction may be parametrically smaller than this estimate. The reason is that for certain sets of parameters the corresponding 1D models are integrable. One example is provided by $S U(2)$ symmetric spinor liquid of bosons or fermions with point-like interactions [16], where the bare masses and interaction constants of both spin species are the same: $M=m$ and $G=g$ [17. The other example is the Tonks gas $g \rightarrow \infty$ with $M=m$ and an arbitrary interaction constant $G$ between the spin excitation and the liquid [18, 19]. As we explicitly verify here in such integrable cases the dissipation is absent [20] and the spin excitations have infinite life-time even at finite temperature. Thus in the vicinity of integrable points the dissipation is strongly suppressed (see, e.g., Ref. 21] for experimental evidence in the spinless case).

In a generic case we found that the friction coefficient may be expressed through the thermodynamic properties of the gas. To this end one needs to know the chemical potentials of the majority $\mu(n)$ and minority $\mu_{d}(n)$ spin species as functions of majority spins concentration $n$ (the minority concentration is vanishingly small). For the friction coefficient at small velocity, $V \ll c$, we found

$$
\begin{aligned}
\kappa & =\frac{16 \pi^{3}}{15} \frac{T^{4}}{c^{4} \hbar^{3} n^{2}}\left[1-\delta_{l}-\frac{M}{m}\right. \\
& \left.+\frac{m}{M^{*}}\left(1-\delta_{l}-\frac{M}{m}\right)^{2}+\frac{\alpha n^{2}}{m c^{2}}\left(1-\delta_{l}-\frac{\alpha_{d}}{\alpha}\right)\right]^{2}
\end{aligned}
$$

Since the effective mass of the spin excitations in the
strong coupling regime is large $M^{*} \gg M[7]$, its velocity
is small $V \ll c$ and the friction coefficient is practically

Since the effective mass of the spin excitations in the
strong coupling regime is large $M^{*} \gg M[7]$, its velocity
is small $V \ll c$ and the friction coefficient is practically

Since the effective mass of the spin excitations in the
strong coupling regime is large $M^{*} \gg M[7]$, its velocity
is small $V \ll c$ and the friction coefficient is practically

where the sound velocity is related to the compressibility as $m c^{2} / n=\partial \mu / \partial n$ and $\alpha=\partial^{2} \mu / \partial n^{2}$, while

$$
\frac{m c^{2}}{n}\left(1-\delta_{l}\right)=\frac{\partial \mu_{d}}{\partial n} ; \quad \alpha_{d}=\frac{\partial^{2} \mu_{d}}{\partial n^{2}} .
$$

In the $S U(2)$ symmetric case the equality of bare masses and interaction constants imply $\delta_{l}=0$ and $\alpha_{d}=\alpha$. As a result the friction is absent $\kappa=0$. The same is true for the $M=m$ Tonks gas, which is equivalent to an impurity moving through the non-interacting Fermi gas 22]. Finding the chemical potentials and the effective mass for non-integrable cases is a difficult task. Analytic

Weak coupling limit: $g \ll \hbar^{2} n / m$ and $G \ll \hbar^{2} n / M$. According to Bogoliubov theory the mean-field equations of state are $\mu=g n$ and $\mu_{d}=G n$, while $M^{*} \simeq M$ [7]. Extracting parameters as in Eq. (4) for both spin excitation and the liquid yields

$$
\kappa(0)=\frac{16 \pi^{3}}{15} \frac{T^{4}}{c^{4} \hbar^{3} n^{2}}\left(\frac{G}{g}\right)^{2}\left(\frac{m G}{M g}-1\right)^{2} .
$$

Keeping the velocity dependence of the friction force, one finds $\kappa(V)=\kappa(0) /\left(1-V^{2} / c^{2}\right)$. As a result, the drift velocity of the spin excitations cannot surpass the speed of sound, $V_{\text {drift }} \leq c$, making it hard to observe Bloch oscillations in weakly interacting gases.

Strong coupling limit: (i) $M \neq m$ and $g, G \rightarrow \infty$. Both majority and minority spins may be considered as two free Fermi gases separated by impermeable mobile wall. Demanding the equal pressure of the two gases, one may find their total kinetic energy and thus respective chemical potentials: $\mu=(\pi \hbar n)^{2} /(2 m)$ and $\mu_{d}=(m / M)^{1 / 3} \mu$. Substituting it in Eqs. (3), (4) and employing that in this regime the effective mass is large $M^{*} / M \sim G M / \hbar^{2} n$, one finds

$$
\kappa=\frac{16 \pi^{3}}{15} \frac{T^{4}}{c^{4} \hbar^{3} n^{2}}\left[\left(\frac{m}{M}\right)^{1 / 3}-\frac{M}{m}\right]^{2} .
$$

(ii) $M=m$ and $g, G \gg \hbar^{2} n / m$. In this case we treat the corresponding Fermi gases as being weakly interacting and find leading perturbative corrections to the chemical potentials: $\mu=\left(\pi^{2} \hbar^{2} / 2 m\right)\left(n^{2}-16 \hbar^{2} n^{3} / 3 g m\right)$, while $\mu_{d}=\left(\pi^{2} \hbar^{2} / 2 m\right)\left(n^{2}-4 \hbar^{2} n^{3}[1 / g+1 / 3 G] / m\right)$. Inserting it in the Eq. (3), one finds that in this order of expansion all the terms cancel each other. Such a cancelation is not expected if the order $n^{4}$ is kept in the chemical potentials. One can thus estimate the friction coefficient as

$$
\kappa \sim \frac{\pi^{3} T^{4}}{c^{4} \hbar^{3} n^{2}}\left(\frac{\hbar^{2} n}{g m}\right)^{4}\left(1-\frac{g}{G}\right)^{2} .
$$
progress may be achieved in two limits: 
velocity-independent. It is in this regime where the Bloch oscillations are most likely to be observed.

We turn now to the derivation of Eq. (3). If the temperature is less than the chemical potential $T \ll \mu$ the density excitations of the majority spin gas may be described [23] by the effective 1D hydrodynamic Hamiltonian

$$
H_{\mathrm{ph}}=\int d x\left[\frac{1}{2 m}(n+\rho)\left(\partial_{x} \vartheta\right)^{2}+\frac{m c^{2}}{2 n} \rho^{2}+\frac{\alpha}{3 !} \rho^{3}\right],
$$

hereafter $\hbar=1$. Here $\rho(x)$ is the operator of density fluctuations on top of the uniform density $n$ and its canonically conjugate phase operator $\vartheta(x)$ is related to the superfluid velocity $v_{s}=\partial_{x} \vartheta / m$. The quadratic (Luttinger liquid) part of this Hamiltonian describes phonons with the linear dispersion relation $\omega(q)=c|q|$. To have a consistent description of the interactions between phonons and the spin excitations, one needs to take into account non-linear interactions of phonons between themselves [5]. They are described by the terms $\sim \rho\left(\partial_{x} \vartheta\right)^{2}$ and $\sim \rho^{3}$. The coefficients in front of them are dictated by Galilean invariance for the former, and by expansion of $\mu(n+\rho(x))$ up to the second order in $\rho$ for the latter.

The spin excitation may be thought of as a quantum particle described by the canonically conjugated coordinate $X$ and momentum $P$. Its interactions with the density fluctuations are encoded in the chemical potential $\mu_{d}(n+\rho(X)) \approx\left(m c^{2} / n\right)\left(1-\delta_{l}\right) \rho(X)+\left(\alpha_{d} / 2\right) \rho^{2}(X)$, cf. Eq. (4). The interactions of this particle with the superfluid velocity $v_{s}(X)$ may be found [5] by noticing that in the reference frame where $v_{s}=0$ the energy of the particle with momentum $P$ is given by the dispersion relation $\varepsilon(P)$. In the laboratory frame momentum of such particle is $P+M v_{s}$, while its energy is $\varepsilon_{v_{s}}\left(P+M v_{s}\right)=\varepsilon(P)+P v_{s}+M v_{s}^{2} / 2$. Changing to a momentum in the laboratory frame, and keeping terms up to the second power in $v_{s}$, one finds $\varepsilon_{v_{s}}(P)=$ $\varepsilon(P)+(P-M V) v_{s}+\left(M^{2} \varepsilon^{\prime \prime}(P)-M\right) v_{s}^{2} / 2$, where the particle velocity is $V=\varepsilon^{\prime}(P)$. At a sufficiently small velocity $V=P / M^{*}$ and $\varepsilon^{\prime \prime}(0)=1 / M^{*}$ one thus finds for the Hamiltonian of the spin excitations

$H_{d}=\frac{\left(P+\delta M v_{s}\right)^{2}}{2 M^{*}}-\frac{\delta M v_{s}^{2}}{2}+\frac{m c^{2}}{n}\left(1-\delta_{l}\right) \rho+\frac{\alpha_{d}}{2} \rho^{2},(9)$

where $\delta M=M^{*}-M$. In the absence of interactions $\delta M=M^{*}-M=0$ and Hamiltonian (9) is independent of the fluid velocity. The terms containing $\rho=\rho(X)$ and $v_{s}=\partial_{x} \vartheta(X) / m$ can be regarded as effective interaction potential dependent on the particle coordinate $X$. This introduces a preferential frame for the moving particle.

It is convenient to perform canonical transformation of the particle momentum $P+\delta M v_{s} \rightarrow P$ along with the fluid "coordinate" $\rho(x)-(\delta M / m) \rho_{d}(x) \rightarrow \rho(x)$, where $\rho_{d}(x)=\delta(x-X)$ is the density of the particle. The changes induced by this transformation to the fluid
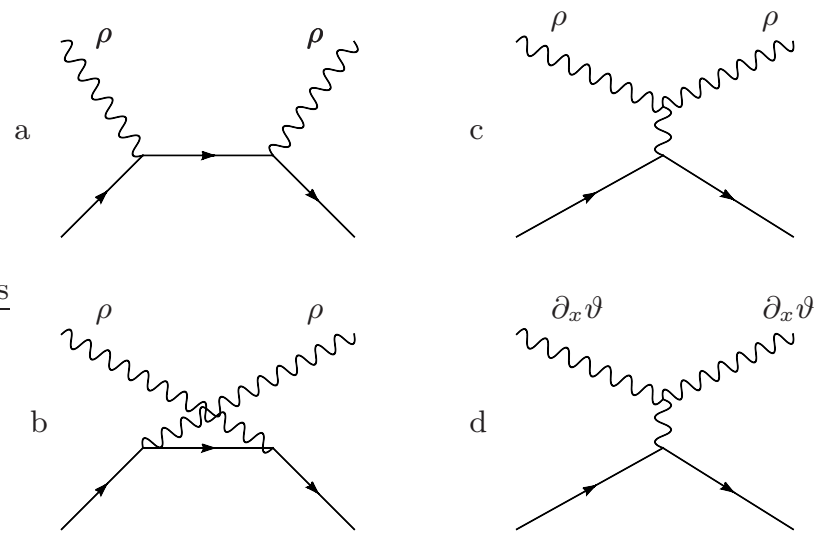

FIG. 2: Second order diagrams contributing to 2-phonon amplitudes. Spin excitation is represented by a full line, while phonons by wavy lines. Diagrams a,b and c contribute to $\Gamma_{\rho}$, Eq. (12), while diagram d represents $\Gamma_{\vartheta}$, Eq. (13).

Hamiltonian, Eq. (8), are absorbed in the modified impurity Hamiltonian

$H_{d}=\frac{P^{2}}{2 M^{*}}+\frac{m c^{2}}{n}\left(1-\delta_{l}+\frac{\delta M}{m}\right) \rho+\frac{\alpha}{2}\left(\frac{\alpha_{d}}{\alpha}+\frac{\delta M}{m}\right) \rho^{2}$.

The second term here describes processes in which one phonon is absorbed or emitted, while the third one is responsible for the two-phonon processes. Due to the quadratic dispersion relation of the spin excitation and linear dispersion of phonons, the one-phonon processes do not lead to dissipation, which is just another statement of Landau criterion.

We thus focus on the two-phonon amplitude. The latter originates from the last term in Eq. (10) as well as the following second order processes: (i) second order in $\rho(X)=\int d x \rho(x) \rho_{d}(x)$ interaction vertex, Fig. 2a,b; (ii) first order in $\rho \rho_{d}$ and first order in $\rho^{3}$, Fig. 2k or $\rho\left(\partial_{x} \vartheta\right)^{2}$ phonon nonlinearity vertexes, Fig. 2d. It is the destructive interference of these second order processes which is responsible for the partial or even complete suppression of the dissipation. Evaluating the corresponding diagrams according to the standard rules, one derives an effective Hamiltonian of the spin excitation 24]

$$
H_{d}^{\mathrm{eff}}=\frac{P^{2}}{2 M^{*}}-\frac{1}{2} \Gamma_{\rho}[\rho(X)]^{2}-\frac{1}{2} \Gamma_{\vartheta}\left[\partial_{x} \vartheta(X)\right]^{2},
$$

where the effective two-phonon amplitudes are given by

$$
\begin{aligned}
& \Gamma_{\rho}=\frac{m^{2} c^{2}}{n^{2} M^{*}}\left(1-\delta_{l}+\frac{\delta M}{m}\right)^{2}-\alpha\left(\frac{\alpha_{d}}{\alpha}-1+\delta_{l}\right) \\
& \Gamma_{\vartheta}=\frac{1}{m}\left(1-\delta_{l}+\frac{\delta M}{m}\right)
\end{aligned}
$$

The momentum relaxation rate may be evaluated in the second order in the two-phonon amplitudes $\Gamma_{\rho, \vartheta}$, averaged over the Luttinger (quadratic) part of the phonon 
Hamiltonian (8). This does not assume smallness of the amplitudes $\Gamma_{\rho, \vartheta}$, but rather gives the leading lowtemperature, $T \ll \mu$, contribution. The semiclassical equation of motion for the spin excitation acquires a form

$$
\dot{P}=-\frac{1}{4}\left(\Gamma_{\rho}-\frac{m^{2} c^{2}}{n^{2}} \Gamma_{\vartheta}\right)^{2} \int \frac{d q}{2 \pi} q \Pi(q, q V),
$$

where $\Pi(q, \omega)$ is the imaginary part of the Fourier transform of $\theta(t)\left\langle\left[\rho^{2}(x, t), \rho^{2}(0,0)\right]\right\rangle$ response function of the phonon gas. The latter is given by

$$
\Pi(q, \omega)=\frac{n^{2}}{4 m^{2} c^{3}}\left(q^{2}-\frac{\omega^{2}}{c^{2}}\right)\left(\operatorname{coth} \frac{c q-\omega}{4 T}-\operatorname{coth} \frac{c q+\omega}{4 T}\right) .
$$

For spin excitations of small velocity, $V \ll c$, one finds

$$
\Pi(q, q V)=\frac{n^{2}}{8 m^{2} c^{3}} \frac{q^{3}}{T} \frac{V}{\sinh ^{2}(c q / 4 T)} .
$$

Substituting Eqs. (12), (13) and (16) in Eq. (14), one finds the friction force $\dot{P}=-\kappa V$ with the friction coefficient $\kappa$ given by Eq. (3). Notice that in the integrable $S U(2)$ symmetric case the two-phonon amplitudes $\Gamma_{\rho}=M^{*} c^{2} / n^{2}$ and $\Gamma_{\vartheta}=M^{*} / m^{2}$ are finite, while the dissipation rate vanishes due to the interference of the density and current excitations, cf. Eq. (14).

Finally we give some estimates for the critical force $F_{c}$. For ${ }^{87} \mathrm{Rb}$ gas with the linear density $n=10^{5} \mathrm{~cm}^{-1}$ and the $1 \mathrm{D}$ interaction constant $g$ of the same order as $\hbar^{2} n / m$, one estimates the sound velocity as $c \approx 1.4 \hbar n / m \approx$ $1 \mathrm{~cm} / \mathrm{s}$. Taking $M=m$ and $M^{*} \approx 1.3 m$ [7], and the temperature $T \approx 0.5 m c^{2} \approx 5 \times 10^{-7} \mathrm{~K}$, one finds for the naive critical force $F_{c}^{(0)} \approx \pi^{3} T^{4} /\left(c^{4} \hbar^{2} n M^{*}\right) \approx$ $4 \times 10^{-22} \mathrm{~N}$. For comparison, the gravitational force acting on ${ }^{87} \mathrm{Rb}$ atom is $1.4 \times 10^{-24} \mathrm{~N}$, i.e. about 300 times weaker. However, closeness to the integrability with say $G / g=1.03$ decreases the actual critical force $F_{c}$ by about three orders of magnitude, see Eqs. (5) or (7). This makes the critical force to be well below the gravitational one, making it possible to observe Bloch oscillations of the spin-flipped particle falling in the gravitational field.

We are grateful to L. I. Glazman, M. Köhl and K. Bongs for stimulating discussions. D.M.G. acknowledges support by EPSRC Advanced Fellowship EP/D072514/1 and a warm hospitality of Mathematical Physics group at Lebedev Physical Institute of Russian Academy of Sciences. A.K. was supported by NSF grants DMR-0405212, DMR- 0804266 and acknowledges EPSRC grant GR/T23725/01 for the support of his stay in Birmingham.

[1] H. J. Lewandowski et al., Phys. Rev. Lett. 88, 070403 (2002); J. M. McGuirk et al., Phys. Rev. Lett. 89, 090402 (2002); J. M. McGuirk et al, Phys. Rev. Lett. 91, 150402 (2003); K. M. Mertes et al., Phys. Rev. Lett. 99, 190402 (2007); X. Du et al., Phys. Rev. Lett. 101, 150401 (2008).

[2] E. P. Bashkin, Pis'ma Zh. Eksp. Teor. Fiz. 33, 11 (1981) [JETP Lett. 33, 8 (1981)]; E. P. Bashkin, Zh. Eksp. Teor.
Fiz. 87, 1948 (1984) [Sov. Phys. JETP 60, 1122 (1985)]; C. Lhuillier and F. Laloë, J. Phys. (Paris) 43, 197 (1982); C. Lhuillier and F. Laloë, J. Phys. (Paris) 43, 225 (1982); L. P. Lévy and A. E. Ruckenstein, Phys. Rev. Lett. 52, 1512 (1984).

[3] A. P. Chikkatur et al., Phys. Rev. Lett. 85, 483 (2000).

[4] L. D. Landau and I. M. Khalatnikov, Zh. Eksp. Teor. Fiz. 19, 637 (1949); L. D. Landau and I. M. Khalatnikov, Zh. Eksp. Teor. Fiz. 19, 709 (1949).

[5] G. Baym and C. Ebner, Phys. Rev. 164, 235 (1967).

[6] B. Laburthe Tolra et al., Phys. Rev. Lett. 92, 190401 (2004); B. Paredes et al., Nature 429, 277 (2004); T. Kinoshita T. Wenger and D. S. Weiss, Science 305, 1125 (2004); M. Köhl et al., Appl. Phys. B 79, 1009 (2004).

[7] J. N. Fuchs et al., Phys. Rev. Lett. 95 (2005).

[8] M. B. Zvonarev, V. V. Cheianov, and T. Giamarchi, Phys. Rev. Lett. 99, 240404 (2007).

[9] K. A. Matveev and A. Furusaki, Phys. Rev. Lett. 101, 170403 (2008).

[10] A. Kamenev and L. I. Glazman, arXiv:0808.0479.

[11] A. Lamacraft, arXiv:0810.4163.

[12] T. Donner et al., Science 315, 1556 (2007).

[13] O. Morsch, et al., Phys. Rev. Lett. 87, 140402 (2001).

[14] According to fluctuation-dissipation theorem, the friction force is accompanied by stochastic force $f(t)$ with the correlator $\left\langle f(t) f\left(t^{\prime}\right)\right\rangle=2 \kappa T \delta\left(t-t^{\prime}\right)$. The complete semiclassical description of the particle kinetics is given by the distribution function $\mathcal{P}(X, P)$, satisfying the FokkerPlanck equation. Here we restrict ourselves with the dynamics of the maximum of this distribution only.

[15] A. H. Castro Neto and M. P. A. Fisher, Phys. Rev. B 53, 9713 (1996).

[16] C. N. Yang, Phys. Rev. Lett. 19, 1312 (1967); M. Gaudin, Phys. Lett. A 24, 55 (1967).

[17] Throughout this paper we assume, where necessary, a microscopic model of short range interactions, $g_{a b} \delta(x-y)$ between particles in internal hyperfine states $a, b=1,2$ conveniently described by spin $1 / 2$ degrees of freedom. The interactions within the fluid are proportional to $g=g_{11}$ and interactions with the "spin flipped" impurity particle are proportional to $G=g_{12}$. Relation of $g_{a b}$ to the parameters of the trapped quantum gas can be found in M. Olshanii, Phys. Rev. Lett. 81, 938 (1998).

[18] J. B. Mcguire, J. Math. Phys. 6, 432 (1965); J. Math. Phys. 7, 123 (1966).

[19] H. Castella and X. Zotos, Phys. Rev. B 47, 16186 (1993).

[20] X. Zotos, J. Low. Temp. Phys. 126, 1185 (2002).

[21] T. Kinoshita T. Wenger and D. S. Weiss, Nature 440, 900 (2006).

[22] According to Refs. [18, 19] the chemical potential of an $M=m$ impurity interacting with a free Fermi gas as $G \sum_{i} \delta\left(X-x_{i}\right)$ is $\mu_{d}=\mu-(n G / 2)\left[\left(x+x^{-1}\right) \arctan x-1\right]$, where $x=$ $2 \pi \hbar^{2} n /(m G)$ and $\mu=\pi^{2} \hbar^{2} n^{2} /(2 m)$. The effective mass is $m / M^{*}=(\pi / 2)\left[\arctan x-1 /\left(x+x^{-1}\right)\right] \arctan ^{-2} x$. Substituting these expressions into Eqs. (3), (4), one finds $\kappa=0$.

[23] See, e.g., V.N. Popov, Functional integrals and collective excitations (Cambridge University Press, 1987).

[24] There is also a cross-term $-\Gamma_{\rho \vartheta} \rho(X) \partial_{x} \vartheta(X)$, where $\Gamma_{\rho \vartheta}=V\left(1-\delta_{l}+\delta M / m\right) / n$, but it does not contribute to the momentum relaxation rate. 